\title{
Tyrosine kinase inhibitors in dermatology: A systemic review
}

Piyu Parth Naik

Department of dermatology, Saudi German hospitals and clinics, Hessa Street 331 West, Al Barsha 3, Exit 36 Sheikh Zayed Road, Opposite of American School, Dubai, United Arab Emirates.

*Corresponding Author: Piyu Parth Naik, Department of dermatology, Saudi German hospitals and clinics, Hessa Street 331 West, Al Barsha 3, Exit 36 Sheikh Zayed Road, Opposite of American School, Dubai, United Arab Emirates.

Received date: May 05, 2021; Accepted date: May 21, 2021; Published date: May 24,2021

Citation: Piyu P Naik. (2021) Tyrosine kinase inhibitors in dermatology: A systemic review. J Dermatology and Dermatitis. 6(1); Doi: 10.31579/2578-8949/075

Copyright: (C2021 Piyu Parth Naik, This is an open-access article distributed under the terms of The Creative Commons. Attribution License, which permits unrestricted use, distribution, and reproduction in any medium, provided the original author and source are credited.

\begin{abstract}
:
The inhibition of enzyme subgroups of tyrosine kinases can block the ability of various cytokines at the same time. Tyrosine kinase inhibitors are a class of drugs that can be used either primarily as oral medicines or in dermatological definitions locally. In several skin problems, restraint of tyrosine kinases has been demonstrated to be advantageous. Aim of this article is to explore the role of tyrosine kinase inhibitors in various skin diseases. Tyrosine inhibitors have demonstrated a guaranteed response in psoriasis, alopecia areata, Vitiligo, while the variable response in other skin disorders, including atopic dermatitis, chronic actinic dermatitis, dermatomyositis, erythema multiforme, graftversus-host disease, hypereosinophillic syndrome, STING vasculopathy, palmoplantar pustulosis and lupus. The approach of Tyrosine inhibitors in dermatology has been met with incredible interest. This class of prescriptions can generously propel the treatment of inflammatory and immune mediated dermatoses. It is still too early to make a strong inference regarding these medicines' place in dermatological treatment, even though the evidence provides promising results.
\end{abstract}

Keywords: tyrosine kinase inhibitors; jak inhibitors; alopecia areata; atopic dermatitis; baricitinib; psoriasis; ruxolitinib; tofacitinib; vitiligo

\section{Introduction:}

Our understanding of the pathogenesis of provocative and immunological infections has gained extraordinary advancement over recent years, thus revealing novel remedial goals. Protein tyrosine kinases (PTKs) handle cell multiplication, immune response and bolstering signaling in cells within theoretical models. Unchecked movements from tyrosine kinase receptors and intracellular tyrosine kinases can lead to fiery reactions of cytokines and lead to autoimmune diseases, dermatitis and even malignancy [1].

The Tyrosine kinase inhibitors, necessary for the downstream motioning of inflammatory cytokines, are among late perceived targets [1]. In dermatology, tyrosine kinase inhibitors have their spot in the blockage of cytoplasmic Janus kinases, which is a subclass of huge tyrosine kinase family. Various investigators have thought about aiming Janus kinases or JAKs starting late due to promising initial results. New disclosures in JAK-STAT pathway and the improvement of medications that block their related activity have made new entryways for treating issues related to this system's dysregulation [2].

After clinical fundamentals performed, with first generation of JAK inhibitors like tofacitinib, ruxolitinib, baricitinib and oclacitinib, dermatological conditions like psoriasis and atopic dermatitis, received the dermatology field's most significant advantage [2].

Another condition that has shown promising outcomes from treatment with JAK-inhibitors is Alopecia areata (AA). Other immune mediated cutaneous disorders where JAK-inhibitors are useful include Vitiligo, atopic dermatitis (AD), chronic actinic dermatitis, chronic mucocutaneous candidiasis, cutaneous T-cell lymphoma, dermatomyositis, hypereosinophillic syndrome, cutaneous Graft Versus Host Disease (GVHD), cutaneous mastocytosis, palmoplantar pustulosis, STING vasculopathy, and lupus erythematosus [2].

\section{Study Design}

The current systematic review has followed Preferred Reporting Items for Systematic Reviews (PRISMA) method [3]. A systematic review comprises the structured collection of evidence-based articles followed by systematically discussing their findings based on the review's aim.

\section{Search Strategy}

An articles search was undertaken electronically using online databases such as Google Scholar, MEDLINE, PubMed, Scopus and Cochrane databases with the appropriate key terms (MeSH). We were looking specifically for articles on Tyrosine kinase inhibitors in dermatology. The keywords were changed according to each database's searching protocol, including "Tyrosine kinase inhibitors," "JAK inhibitors," "Dermatology." Bibliographic sources were also screened.

\section{Inclusion and exclusion criteria}

The articles published related to Tyrosine kinase inhibitors in dermatology were included in this review. The research articles, case studies, studies related to other than dermatological disorders, review and 
systematic review and articles published other than the English language were excluded in this study.

\section{Data extraction}

The initial literature search revealed 717 articles. Relevant articles were chosen for full-text screening after the application of the eligibility criteria.

\section{Eligible studies}

The literature search yielded 717 articles from various databases, including PubMed, Ovid, Science Direct and Google Scholar, of which 305 articles were excluded at the initial stage due to repetition and irrelevance. Out of 412, 278 articles were further excluded after analyzing the titles and abstracts at the first screening level. Finally, 134 studies meeting the current systematic review's inclusion criteria as detailed in the PRISMA flow chart were included in this review. (Figure 1)
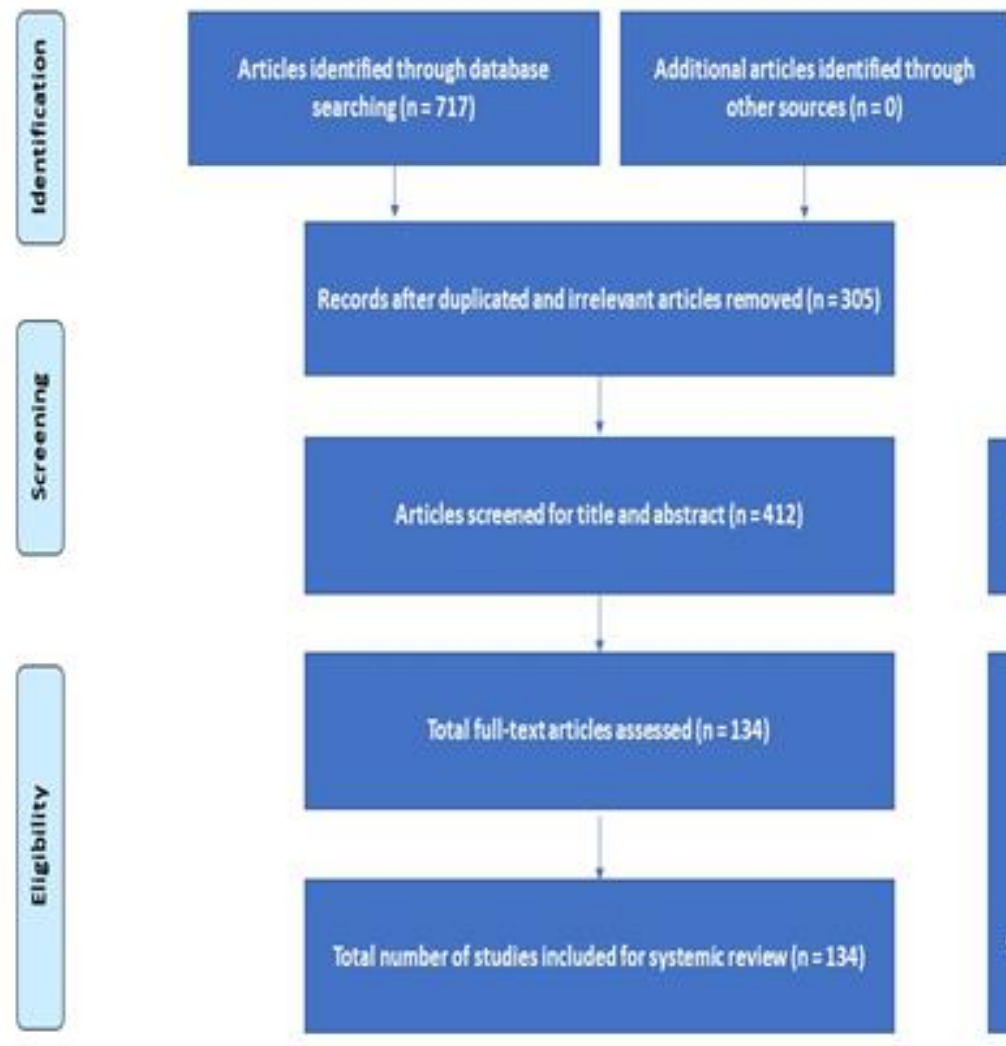

Artides excluded after reading the

abstract as irrelevart, sresentabians

and other disease $(n=278)$
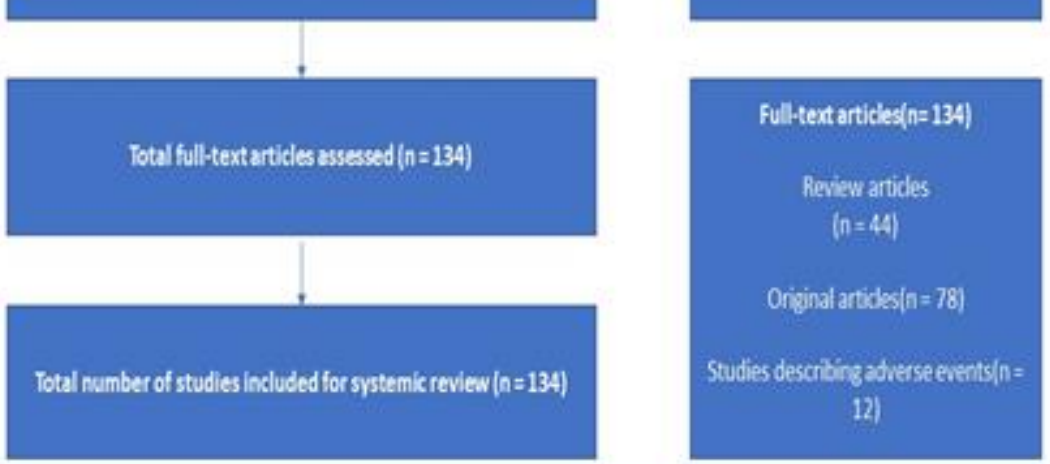

Figure 1: PRISMA flow chart

The references to each relating articles have additionally been looked up. Furthermore, clinical trials were further used to look for clinical preliminaries.

Our hunt yielded 134 items, comprising 44 review articles, 78 unique original articles (53 clinical investigations and 25 preclinical examinations in vitro and in vivo), and 12 reports on adverse events.

\section{Results}

Among the clinical examinations (43\%) and clinical trials $(38.7 \%)$, Psoriasis was the most well-known skin disease found, which responded to this group of medicines. The other common condition considered in preclinical investigations was dermatitis (Table 1).

\begin{tabular}{|c|c|c|}
\hline $\begin{array}{c}\text { Preclinical studies } \\
n=25(\%)\end{array}$ & $\begin{array}{c}\text { Clinical studies } \\
n=53(\%)\end{array}$ & $\begin{array}{c}\text { Clinical trials, } \mathrm{n}=18 \\
(\%)\end{array}$ \\
\hline Dermatitis, 7 (28\%) & $\begin{array}{c}\text { Psoriasis, } 23(43 \%), 1 \text { with } \\
\text { psoriatic arthritis }\end{array}$ & Psoriasis, $4(22 \%)$ \\
\hline CTCL/Sezary syndrome, $6(24 \%)$ & AA, $14(26 \%), 2$ with psoriasis & Psoriatic, arthritis, $3(16.7 \%)$ \\
\hline GVHD, $5(20 \%)$ & GVHD, $4(7.5 \%)$ & GVHD, $3(16.7 \%)$ \\
\hline Psoriasis, $3(12 \%)$ & CMC,2 (4\%), 1 with AA & AA, $2(11.1 \%)$ \\
\hline Psoriatic arthritis, $1(4 \%)$ & Vitiligo, 2 (4\%), 1 with AA & Atopic dermatitis, $2(11.1 \%)$ \\
\hline AA, $1(4 \%)$ & Dermatomyositis, $2(4 \%)$ & Systemic LE, $2(11.1 \%)$ \\
\hline Systemic and cutaneous LE, $1(4 \%)$ & LE, $2(4 \%)$ & CANDLE, JDM and SAVI, $1(0.06 \%)$ \\
\hline Skin aging, $1(4 \%)$ & $\begin{array}{c}\text { Atopic dermatitis, } 2(4 \%) \\
\text { SAVI, } 2(4 \%)\end{array}$ & Vitiligo, $1(0.06 \%)$ \\
\hline
\end{tabular}

Table 1: Studies of Janus Kinase inhibitors for common cutaneous diseases found through systematic review. 
The adequacy of Tyrosine Kinase inhibitors in the treatment of skin and hair problems was accounted for in 29 of the 46 papers recognized.

\section{Discussion}

The pathway of tyrosine kinase and associated cytokines to action and dysregulation has been connected as a potential psoriasis disease component. Either oral or topically administered tyrosine kinase inhibitors have been beneficial in the treatment of Psoriasis. The most extensively read finished paperwork for Tyrosine Kinase inhibitors in dermatology have been in the subject of Psoriasis to date.

The efficacy of oral tofacitinib was studied in chronic plaque psoriasis in phase 3 randomized controlled trials by Papp et al. in 2015, which showed noteworthy effectiveness in patients with moderate-to-severe psoriasis [4]. In another assessment by Bachelet et al., the Psoriasis Area Severity Index (PASI) responded to oral tofacitinib by $39.5 \%$ in $5 \mathrm{mg}$ dose group and $63.6 \%$ in $10 \mathrm{mg}$ dose group at 12 weeks [5]. Tofacitinib in $10 \mathrm{mg}$ dosage was non-inferior to etanercept therapy in moderate-severe cases of plaque psoriasis [5]. For the $5 \mathrm{mg}$ and $10 \mathrm{mg}$ divided frameworks of tofacitinib, adversarial events were similar for tofacitinib and etanercept [5]. Similar improvement was also observed in another study done by Boy et al [6]. However, FDA has not yet approved tofacitinib for psoriasis and its role still remains investigational till date.

Vesely et al. observed substantial improvement in a refractory, severe case of chronic actinic dermatitis to tofacitinib [7]. Infect, same patient was put on multiple immunomodulatory agents prior to tofacitinib administration, which shows zenith of JAK inhibitors, especially tofacitinib [7].

Role of tofacitinib in cutaneous manifestations of polyarteritis nodosa (PAN) was highlighted by Rimar et al. in 2016 [8]. A young adult with chronic symptoms of livedo reticularis and skin nodules, previously treated with azathioprine and methotrexate in remission when showed flaring up of disease showed response to tofacitinib [8].

Seventeen examinations were assessed for the utilization of tyrosine kinase inhibitors in AA. Harel et al observed that local treatment of human and mouse skin with JAK-inhibitors culminated in swift transition of anagen phase of hair cycle and hence hair growth [9]. Core pathogenesis of alopecia areata by interferon gamma and gamma chain cytokine receptors was studied by Xing et al., which also demonstrated the positive role of JAK inhibitors in hair regrowth and turnaround of entrenched AA [10]. These outcomes can clarify the rationale and benefits of tyrosine kinase inhibitors therapy in patients with AA. Sixty-six patients with AA treated by tofacitinib were assessed by Crispin et al. in open -label study in 2016. The impact of tofacitinib was significant. In 32 percent of patients, significant improvement was illustrated in form of regrowth of scalp hair. [11].

Another investigation, including 90 AA patients treated with Tofacitinib, announced clinical response in $77 \%$ of cases. In this particular study, response in AA was more marked in comparison to alopecia totalis or alopecia universalis [12]. Additionally, Craiglow et al. displayed the encouraging role of tofacitinib in teenage group cases of AA [13].

In a pilot study, ruxolitinib was given to 12 patients of moderate-to-severe alopecia areata. Striking hair regrowth was seen in 9 patients after 3 to 6 months of treatment with ruxolitinib [14]. Additional investigation on eyebrow and scalp with ruxolitinib brought complete eyebrow hair regrowth and $10 \%$ of scalp hair regrowth [15].

Study done by Jabbari et al. illustrated that another tyrosine kinase inhibitor named Baricitinib may be fruitful treatment of AA [16]. Same Baricitinib also attained remarkable amelioration in moderate-to-severe patients of psoriasis and PASI-75 showed revamp after 12 weeks of treatment $[17,18]$.

JAK inhibitor, tofacitinib showed marked repigmentation in a case of vitiligo, which was attributed to modern discovery in pathogenesis of vitiligo [19]. Similar efficacy of oral ruxolitinib was visualized in a case of coexistent vitiligo and AA by Harris et al [20]. Recent clinical trial by Rosmarin et al. showed successful repigmentation of vitiligo patches with ruxolitinib cream [21]

Reduction in the overall surface area of dermatitis with associated reduction of excoriation and erythema as well as lichenification was detected after Oral tofacitinib usage in recalcitrant atopic dermatitis with $66.6 \%$ reduction in $\mathrm{AD}$ index [22]. Bissonnette et al. noticed pronounced effectiveness of tofacitinib ointment for atopic dermatitis in a randomized trial [23]. They even considered topical route propitious in atopic dermatitis cases [23].

Ruxolitinib was triumphant against a difficult-to-treat case of chronic mucocutaneous candidiasis as reported by Mossner et al. with IL- 6 being used as feedback variable [24].

Cutaneous $\mathrm{T}$ cell lymphoma are a subgroup which comes under the broad heading of non-Hodgkin lymphoma which harbors mutations in $\mathrm{T}$ cell receptor complex and JAK-STAT signaling pathways. These collectively make tyrosine kinase inhibitors exciting novel weapon against cutaneous $\mathrm{T}$ cell lymphoma. However, this prospective functionality needs future trials [25].

Kurtzman et al. delineated foremost utilization of tofacitinib in treatment of refractory dermatomyositis [26]. Likewise, revocation of recalcitrant dermatomyositis was discovered by Hornung et al., when Ruxolitinib was dispensed in a case of myelofibrosis where dermatomyositis was associated finding [27].

Tofacitinib treatment in $10 \mathrm{mg}$ dosage led to full recovery in a case of idiopathic recurrent erythema multiforme as stated by Damsky et al [28] A multicenter survey exhibited the positive role of ruxolitinib in steroid resistant cutaneous findings of graft-versus-host disease after stem cell transplantation [29]. Reduction in blood eosinophilic count as well as improvement in dermatitis and itching was depicted after treatment with JAK inhibitors in hypereosinophillic syndrome with dermatological manifestations [30].

Chilblain lupus erythematosus, which is a rare autoimmune disorder, was also controlled by ruxolitinib treatment as stated by Wenzel et al [31]. Ruxolitinib also improves quality of life and symptoms in systemic mastocytosis [32]. Even rarer STING vasculopathy which is linked with TMEM1J3 mutation, improved with ruxolitinib in three children [33]. Koga et al. reported therapeutic favorable outcome in patients of palmoplantar pustulosis treated by JAK inhibitors [34].

Thus, Tyrosine kinase inhibitors are now a major class of drugs in dermatology treating a plethora of cutaneous conditions. (Table 2) 


\begin{tabular}{|c|c|c|}
\hline Agent & Targeted JAK & Tested Dermatologic diseases \\
\hline Tofacitinib & JAK1/3 & psoriasis [5,6], \\
& & alopecia areata [9,11,12,13], \\
& & atopic dermatitis [22, 23], \\
& chronic actinic dermatitis [7], \\
& dermatomyositis [26], \\
& & erythema multiforme [28], \\
& & hypereosinophillic syndrome [30], \\
& & STING vasculopathy [33], \\
& & palmoplantar pustulosis [34], \\
& & vitiligo [19], \\
& & Cutaneous T cell lymphoma [25] \\
& JAK1/2 & systemic lupus erythematosus [31]. \\
\hline Ruxolitinib & & alopecia areata [14,15], \\
& & Vitiligo [20,21], \\
& & Mucocutaneous candidiasis [24] \\
& & systemic lupus erythematosus [31], \\
& & systemic mastocytosis [32], \\
& dermatomyositis [27] and \\
& & GVHD [29] \\
\hline Baricitinib & JAK1/2 & Psoriasis [16,17,18] \\
\hline
\end{tabular}

Table 2: Characteristics of Janus Inhibitors that are most commonly studied for the treatment of dermatologic diseases.

\section{Safety statistics}

Though tyrosine kinase inhibitors have many applications in the vast field of dermatology, this group is not free of side effects. Though tyrosine kinase inhibitors used in clinical oncology have predominant skin and cardiovascular toxicities, TKI which are having skin applications do not demonstrate them [35-38].

Ruxolitinib was most commonly associated with urinary tract infection when it was used in treating myelofibrosis [39]. On the other hand, tofacitinib increased the risk of reactivation stage of varicella zoster virus [40]. Response to vaccination could be impeded with tofacitinib and ruxolitinib, which requires healthcare strategist to plan vaccinations before starting treatment with these tyrosine kinase inhibitors [41].

As JAK2 inhibition is more profound with ruxolitinib as compared with tofacitinib, cytopenia, which is primarily mediated by JAK2, is more common with ruxolitinib [42]. However, in a study carried out by Mackay-Wiggan et al. AA dealt with ruxolitinib in therapeutic doses did not demonstrate cytopenia [14].

Neoplasia risk is definitely a reason of apprehension among scientists with the use of Tyrosine kinase inhibitors, albeit theoretically. $1 \%$ of renal transplant patients treated with tofacitinib developed post-transplant lympho-proliferative disorder [43]. Nevertheless, no obvious malignancy risk was perceptible when tofacitinib or ruxolitinib were used to treat inflammatory diseases till date [44-47].

\section{Conclusions:}

Tyrosine inhibitors have demonstrated response in multiple skin disorders, including Psoriasis, alopecia areata and Vitiligo. Clinical information indicates that the treatment of numerous other dermatological conditions can be achieved through tyrosine hindrance. The approach of Tyrosine inhibitors in dermatology has been met with incredible interest. This class of prescriptions can generously propel the treatment of inflammatory and immune mediated dermatoses. Another exhilarating age of JAK inhibitors/ tyrosine kinase inhibitors is in progress.

\section{Reference:}

1. Gadina M. (2014) Advances in kinase inhibition: treating rheumatic diseases and beyond. CurrOpinRheumatol. 26:237-43. 10 .
2. Damsky W, King BA. (2017) JAK inhibitors in dermatology: The promise of a new drug class. J Am Acad Dermatol; 76(4):736-744.

3. Page MJ, McKenzie JE, Bossuyt PM, Boutron I, Hoffmann TC, Mulrow CD, et al. (2021) The PRISMA 2020 statement: An updated guideline for reporting systematic reviews. PLoS Med 18(3).

4. Papp KA, Menter MA, Abe M, et al. (2015) Tofacitinib, an oral Janus kinase inhibitor, for treating chronic plaque psoriasis: results from two randomized, placebo-controlled, phase III trials. Br J Dermatol; 173(4):949-961.

5. Bachelet H, van de Kerkhof PC, Strohal R, et al. (2015) tofacitinib versus etanercept or placebo in moderate-to-severe chronic plaque psoriasis: a phase 3 randomized non-inferiority trial. Lancet; 386(9993):552-561.

6. Boy MG, Wang C, Wilkinson BE, et al. (2009) Double-blind, placebo-controlled, dose-escalation study to evaluate the pharmacologic effect of CP-690,550 in patients with Psoriasis. J Invest Dermatol; 129:2299-2302.

7. Vesely MD, Imaeda S, King B. (2016) Tofacitinib citrate to treat refractory, severe chronic actinic dermatitis. JAAD Case Rep; 3(1):4-6.

8. Rimar D, Alpert A, Starosvetsky E, et al. (2016) tofacitinib for polyarteritis nodosa: a tailored therapy. Ann Rheum Dis;75:22142216

9. Harel S, Higgins CA, Cerise JE, et al. (2015) Pharmacologic inhibition of JAK-STAT signaling promotes hair growth. Sci Adv; 1: e1500973.

10. Xing L, Dai Z, Jabbari A, et al. (2014) Alopecia areata is driven by cytotoxic $\mathrm{T}$ lymphocytes and is reversed by JAK inhibition. Nat Med; 20:1043-1049.

11. Crispin MK, Ko JM, Craiglow BG, et al. (2016) safety and efficacy of the JAK inhibitor tofacitinib citrate in patients with alopecia areata. JCI Insight; 1:e89776.

12. Liu LY, Craiglow BG, Dai F, King BA. (2017) Tofacitinib for the treatment of severe alopecia areata and variants: a study of 90 patients. J Am Acad Dermatol; 76:22-28.

13. Craiglow BG, Liu LY, King BA. (2017) Tofacitinib for the treatment of alopecia areata and variants in adolescents. J Am Acad Dermatol; 76:29-32. 
14. Mackay-Wiggan J, Jabbari A, Nguyen N, et al. (2016) Oral ruxolitinib induces hair regrowth in patients with moderate-tosevere alopecia areata. JCI Insight; 1:e89790

15. Craiglow BG, Tavares D, King BA. (2016) Topical ruxolitinib for the treatment of alopecia Universalis. JAMA Dermatol; 152: 490491.

16. Jabbari A, Dai Z, Xing L, et al. (2015) Reversal of alopecia areata following treatment with the JAK1/2 inhibitor baricitinib. EBioMedicine; 2:351-355.

17. Papp K, Pariser D, Catlin M, et al. (2015) A phase 2a randomized, double-blind, placebo-controlled, sequential dose-escalation study to evaluate the efficacy and safety of ASP015K, a novel Janus kinase inhibitor, in patients with moderate-to-severe Psoriasis. Br J Dermatol; 173:767-776.

18. Papp KA, Menter MA, Raman M, et al. (2016) A randomized phase $2 b$ trial of baricitinib, an oral Janus kinase (JAK) 1/JAK2 inhibitor, in patients with moderate-to-severe Psoriasis. $\mathrm{Br} \mathbf{J}$ Dermatol; 174:1266-1276.

19. Craiglow BG, King BA. (2015) Tofacitinib citrate for the treatment of Vitiligo: a pathogenesis-directed therapy. JAMA Dermatol; 151:1110-1112.

20. Harris JE, Rashighi M, Nguyen N. (2016) Rapid skin repigmentation on oral ruxolitinib in a patient with coexistent vitiligo and alopecia areata (A.A.). J Am Acad Dermatol;74:370 371

21. Rosmarin D, Pandya AG, Lebwohl M, Grimes P, Hamzavi I, Gottlieb AB, Butler K, Kuo F, Sun K, Ji T, Howell MD, Harris JE. (2020) Ruxolitinib cream for treatment of vitiligo: a randomised, controlled, phase 2 trial. Lancet. 396(10244):110120.

22. Levy LL, Urban J, King BA. (2015) Treatment of recalcitrant atopic dermatitis with the oral Janus kinase inhibitor tofacitinib citrate. J Am Acad Dermatol.73 (3):395-399.

23. Bissonnette R, Papp KA, Poulin Y, et al. (2016) Topical tofacitinib for atopic dermatitis: a phase IIa randomized trial. Br J Dermatol; 175:902-911.

24. Mossner R, Diering N, Bader O, et al. (2016) Ruxolitinib induces interleukin 17 and ameliorates chronic mucocutaneous candidiasis caused by STAT1 gain-of-function mutation. Clin Infect Dis; 62(7):951-953.

25. Damsky WE, Choi J. (2016) Genetics of Cutaneous T cell lymphoma: from bench to bedside. Curr Treat Options Oncol; 17(7): 33.

26. Kurtzman DJ, Wright NA, Lin J, et al. (2016) Tofacitinib citrate for refractory cutaneous dermatomyositis: an alternative treatment. JAMA Dermatol; 152:944-945.

27. Hornung T, Janzen V, Heidgen FJ, Wolf D, Bieber T, Wenzel J. (2014) Remission of recalcitrant dermatomyositis treated with ruxolitinib. N Engl J Med; 371(26):2537-2538.

28. Damsky W, King BA. (2016) Idiopathic erythema multiforme: evidence of underlying JAK-STAT activation and successful treatment with tofacitinib. JAAD Case Rep; 2(6):502-504.

29. Zeiser R, Burchert A, Lengerke C, et al. (2015) Ruxolitinib in corticosteroid-refractory graft-versus-host disease after allogeneic stem cell transplantation: a multicenter survey. Leukemia; 29(10):2062-2068.

30. King B, Lee AI, Choi J. (2016) Treatment of hypereosinophilic syndrome with cutaneous involvement with JAK inhibitors tofacitinib and ruxolitinib. J Invest Dermatol.
31. Wenzel J, van Holt N, Maier J, Vonnahme M, Bieber T, Wolf D. (2016) JAK1/2 inhibitor ruxolitinib controls a case of chilblain lupus erythematosus. J Invest Dermatol; 136(6):1281-1283.

32. Yacoub A, Prochaska L. (2016) Ruxolitinib improves symptoms and quality of life in a patient with systemic mastocytosis. Biomark Res; 4:2.

33. Fremond ML, Rodero MP, Jeremiah N, et al. (2016) Efficacy of the Janus kinase 1/2 inhibitor ruxolitinib in the treatment of vasculopathy associated with TMEM173-activating mutations in 3 children. J Allergy Clin Immunol; 136:1752-1755.

34. Koga T, Sato T, Umeda M, et al. (2016) Successful treatment of palmoplantar pustulosis with rheumatoid arthritis, with tofacitinib: impact of this JAK inhibitor on T-cell differentiation. Clin Immunol; 173:147-178.

35. Hartmann JT, Haap M, Kopp HG, Lipp HP. (2009) Tyrosine kinase inhibitors - a review on pharmacology, metabolism and side effects. Curr Drug Metab; 10(5):470-81.

36. Chaar M, Kamta J, Ait-Oudhia S. (2018) Mechanisms, monitoring, and management of tyrosine kinase inhibitorsassociated cardiovascular toxicities. Onco Targets Ther; 11:62276237.

37. Orphanos GS, Ioannidis GN, Ardavanis AG. (2009) Cardiotoxicity induced by tyrosine kinase inhibitors. Acta Oncol; 48(7):964-70.

38. Pottier C, Fresnais M, Gilon M, Jérusalem G, Longuespée R, Sounni NE. (2020) Tyrosine Kinase Inhibitors in Cancer: Breakthrough and Challenges of Targeted Therapy. Cancers (Basel); 12(3):731.

39. O'Sullivan JM, McLornan DP, Harrison CN. (2016) Safety considerations when treating myelofibrosis. Expert Opin Drug Saf; 15:1185-1192.

40. Winthrop KL, Yamanaka H, Valdez H, et al. (2014) Herpes zoster and tofacitinib therapy in patients with rheumatoid arthritis. Arthritis Rheumatol; 66(10):2675-2684.

41. Winthrop KL, Silverfield J, Racewicz A, et al. (2016) The effect of tofacitinib on pneumococcal and influenza vaccine responses in rheumatoid arthritis. Ann Rheum Dis; 75(4):687-695.

42. Galli S, McLornan D, Harrison C. (2014) Safety evaluation of ruxolitinib for treating myelofibrosis. Expert Opin Drug Saf; 13(7):967-976

43. Vincenti F, Silva HT, Busque S, et al. (2015) Evaluation of the effect of tofacitinib exposure on outcomes in kidney transplant patients. Am J Transplant; 15(6):1644-16.

44. Wollenhaupt J, Silverfield J, Lee EB, et al. (2014) Safety and efficacy of tofacitinib, an oral Janus kinase inhibitor, for the treatment of rheumatoid arthritis in open-label, longterm extension studies. J Rheumatol; 41(5):837-852.

45. Curtis JR, Lee EB, Kaplan IV, et al. (2016) Tofacitinib, an oral Janus kinase inhibitor: analysis of malignancies across the rheumatoid arthritis clinical development programme. Ann Rheum Dis; 75(5):831-841.

46. Yamanaka H, Tanaka Y, Takeuchi T, et al. (2016) Tofacitinib, an oral Janus kinase inhibitor, as monotherapy or with background methotrexate, in Japanese patients with rheumatoid arthritis: an open-label, long-term extension study. Arthritis Res Ther; 18:34.

47. Verstovsek S, Vannucchi AM, Griesshammer M, et al. (2016) Ruxolitinib versus best available therapy in patients with polycythemia vera: 80-week follow-up from the RESPONSE trial. Haematologica; 101(7):821-829. 
This work is licensed under Creative Commons Attribution 4.0 License

To Submit Your Article Click Here: Submit Manuscript

DOI: $10.31579 / 2578-8949 / 075$
Ready to submit your research? Choose Auctores and benefit from:

* fast, convenient online submission

* rigorous peer review by experienced research in your field

* rapid publication on acceptance

* authors retain copyrights

* unique DOI for all articles

* immediate, unrestricted online access

At Auctores, research is always in progress.

Learn more www.auctoresonline.org/journals/dermatology-anddermatitis-- 